\title{
Variational Monte Carlo calculation of dynamic multipole polarizabilities and van der Waals coefficients of the PsH system
}

\author{
Massimo Mella* \\ Dipartimento di Chimica Fisica ed Elettrochimica, Universita' degli Studi di Milano, via Golgi 19, 20133 Milano, Italy \\ Dario Bressanini ${ }^{\dagger}$ and Gabriele Morosi ${ }^{\ddagger}$ \\ Dipartimento di Scienze Chimiche, Fisiche e Matematiche, Universita' dell'Insubria, via Lucini 3, 22100 Como, Italy
}

(Received 13 May 2000; revised manuscript received 25 August 2000; published 16 January 2001)

\begin{abstract}
The first three dynamic multipole polarizabilities for the ground state of hydrogen, helium, hydride ion, and positronium hydride PsH have been computed using the variational Monte Carlo (VMC) method and explicitly correlated wave functions. Results for the static dipole polarizability by means of the diffusion Monte Carlo method and the finite field approach show the VMC results to be quite accurate. From these dynamic polarizabilities van der Waals dispersion coefficients for the interaction of $\mathrm{PsH}$ with ordinary electronic systems can be computed, allowing one to predict the dispersion energy for the interaction between PsH and less exotic atoms and molecules.
\end{abstract}

DOI: $10.1103 /$ PhysRevA.63.024503

PACS number(s): $36.10 .-\mathrm{k}, 02.70 . \mathrm{Rr}$

While experimentalists rely every day on positrons and positronium atoms (Ps) to collect information about microscopic features of macroscopic systems like solutions, polymers and crystals, much less effort has been devoted to the theoretical understanding of the complex interactions that take place between ordinary matter and positrons. Among the explored avenues of this field, we mention the interest in predicting the stability of classes of compounds like $e^{+} M$ and $M$ Ps [1-9], where $M$ represents an atomic or molecular system, and the calculation of the cross sections in the scattering process of $e^{+}$and Ps on a molecule or an atom [1016].

On the contrary, the evaluation of the interaction energy between $e^{+} M$ or $M$ Ps and a molecule or atom is an almost unexplored issue [17]. We believe this fact is primarily due to the need of a very accurate trial wave function to describe correctly the correlated motions of electrons and positrons. So far, only variational calculations with explicitly correlated Gaussians [1,5] or Hylleraas-type functions [7-9], and the diffusion Monte Carlo (DMC) method [2-4] have shown to be able to adequately recover the correlation energy in positron-containing systems.

Related to the calculation of the interaction energies is the calculation of second-order properties of positron-containing systems, a problem whose surface has been barely scratched in the past [6]. These properties, specifically the dynamic polarizabilities, are strictly related to the van der Waals coefficients that describe the long-range interaction between systems [18], representing a way to tackle the problem of the asymptotic intermolecular interactions. Recently, Caffarel and Hess showed that these properties can be computed by means of quantum Monte Carlo simulations [19] connecting the imaginary-time-dependent dynamics of the unperturbed

\footnotetext{
*Electronic address: Massimo.Mella@unimi.it

†Electronic address: Dario.Bressanini@uninsubria.it

‡Electronic address: Gabriele.Morosi@ uninsubria.it
}

system with the transition probabilities of a reference diffusion process. In this paper we apply a modified version of their method to compute dynamic multipole polarizabilities for $\mathrm{PsH}, \mathrm{H}, \mathrm{He}$, and $\mathrm{H}^{-}$as a way to understand the behavior of these systems when interacting with an external field, and as a first step towards the definition of the interaction potential between PsH and the ordinary matter.

As far as we know, the work by Le Sech and Silvi [6] is the only one reporting calculations on the effect of a constant electric field on PsH. In that work they computed both the static dipole polarizability, 123 a.u., and the behavior of the annihilation rate $\Gamma_{2 \gamma}$ versus the intensity of the field employing explicitly correlated wave functions, numerical integration, and a variation-perturbation approach. As a by-product of our calculations of the potential-energy curve of the $e^{+} \mathrm{LiH}$ system [20], we obtained an estimation of the static dipole polarizability of 49(2) a.u., a value quite different from the one computed by Le Sech and Silvi. Since we believe this difference to be too large to admit an explanation based on the different accuracy of the methods used to compute this value, we plan to solve this puzzle in this paper.

In the method by Caffarel and Hess [19] the frequencydependent second-order correction to the ground-state energy is written as a sum of the two time-centered autocorrelation functions of the perturbing potential $V$

$$
E_{ \pm}^{(2)}(\omega)=-\int_{0}^{\infty} e^{ \pm t \omega} C_{V V}(t) d t
$$

where the autocorrelation function $C_{V V}(t)$ is given by

$$
C_{V V}(t)=\langle V(0) V(t)\rangle_{\Psi_{0}^{2}}-\left\langle V(0)^{2}\right\rangle_{\Psi_{0}^{2}}
$$

Here, $\langle\ldots\rangle \Psi_{0}^{2}$ indicates that the average has to be taken using the Langevin dynamics that samples the square of the exact ground-state wave function of the unperturbed system.

Caffarel and Hess [19] showed that it is possible to compute $C_{V V}(t)$ employing an optimized trial wave function and 
the pure-diffusion Monte Carlo (PDMC) method, an alternative algorithm to the commonly used DMC with branching, where each walker explicitly carries its own weight along all the simulation [21].

In their work on $\mathrm{He}$ and $\mathrm{H}_{2}$, Caffarel et al., [22] reported that the autocorrelation function $C_{V V}(t)$ becomes dominated by the noise at large times, and this fact might be due to the fluctuations of the walker weights that increase during a PDMC simulation, while the value of the autocorrelation function itself becomes smaller. While the second effect is intrinsic to the stochastic method, the first can be reduced employing a more accurate trial wave function that is able to reduce the weight fluctuations. Another possibility, giving up the exactness of the method (i.e., not sampling the exact $\Psi_{0}^{2}$ ), is represented by the sampling of a quite accurate trial wave function without carrying around the weight for each walker, a method we call perturbation theory variational Monte Carlo (PT-VMC). This algorithm can be useful for those systems whose autocorrelation function has a large decaying time, as in the case of $\mathrm{H}^{-}$and PsH. This large decaying time will increase the fluctuations of the carried weights, and hence the statistical noise in the autocorrelation functions in the long-time region.

As a test of the correctness of our computer program and of the accuracy of the method, we computed the first three autocorrelation functions, and hence the dynamic polarizabilities up to the octupolar one, for the two systems $\mathrm{H}$ and $\mathrm{He}$. The analytical forms of the perturbing potentials were taken from Ref. [22]. While for $\mathrm{H}$ we employed the exact groundstate wave function and compared with the analytic values of the multipole polarizability [18], for the $\mathrm{He}$ case we used a 25-term Hylleraas-type wave function optimized by means of the standard energy minimization [23]. We fitted the numerical $C_{V V}(t)$ results of our simulations with a linear combination of three exponential functions

$$
C_{V V}(t) \simeq \sum_{i=1}^{3} a_{i} e^{-\lambda_{i} t}
$$

in order to have an analytical representation of the autocorrelation functions at all the times. Since it is important to reproduce accurately the long-time behavior of $C_{V V}$, the smallest $\lambda_{i}$ in Eq. (3) was independently calculated fitting $\ln \left[C_{V V}\right]$ in the long-time region with a first-order polynomial. This choice was found to improve sensibly the goodness of the total fitting in this time range.

These analytical representations of $C_{V V}$ allow us to compute easily the integrals in Eq. (1) and to obtain simple expressions of $\alpha(\omega)$. The parameters obtained by the fitting procedure are available from the authors upon requests.

For both systems we found excellent agreement of the static polarizabilities ( $\mathrm{H} \alpha_{d i p}=4.495$ a.u., $\alpha_{\text {quad }}$ $=15.034$ a.u., $\alpha_{\text {oct }}=133.105$ a.u.; He $\alpha_{d i p}=1.382$ a.u., $\alpha_{\text {quad }}=2.401$ a.u., $\alpha_{\text {oct }}=10.367$ a.u.) with the exact results for H [18], with PDMC results by Caffarel et al. [22], with Glover and Weinhold upper and lower bounds for $\mathrm{He}$ [24], and with the accurate results by Yan et al. [25].

At this point we would like to stress that, although in the PT-VMC method the walkers carry always a unitary weight
TABLE I. Mean values for observables of the ground-state ${ }^{1} S$ of $\mathrm{H}^{-}$. All values are in atomic units.

\begin{tabular}{lcc}
\hline \hline & VMC $^{\mathrm{a}}$ & Hylleraas $^{\mathrm{b}}$ \\
\hline$\langle E\rangle$ & $-0.52701(2)$ & $-0.52775^{\mathrm{b}}$ \\
$\langle V\rangle$ & $-1.0448(2)$ & $-1.0555^{\mathrm{b}}$ \\
$\left\langle r_{-}\right\rangle$ & 2.7262 & $2.7102^{\mathrm{b}}$ \\
$\left\langle r_{-}^{2}\right\rangle$ & 11.844 & $11.915^{\mathrm{b}}$ \\
$\left\langle r_{--}\right\rangle$ & 4.4119 & $4.4127^{\mathrm{b}}$ \\
$\left\langle r_{--}^{2}\right\rangle$ & 24.957 & $25.20^{\mathrm{b}}$ \\
$\alpha_{\text {dip }}$ & 189.30 & $206(3)^{\mathrm{c}}$ \\
$\alpha_{\text {quad }}$ & 5761.5 & \\
$\alpha_{\text {oct }}$ & 450758 & \\
\hline \hline
\end{tabular}

${ }^{\mathrm{a}}$ This paper (five term wave function).

${ }^{\mathrm{b}}$ Reference [26].

${ }^{\mathrm{c}}$ Reference [24].

because the branching process is absent, similarly to the PDMC method the time step has to be chosen short enough to produce only a small time step bias. For these two systems we found the time step of 0.01 hartree $^{-1}$ to be adequate to compute statistically exact results.

As a check of the ability of the PT-VMC method to also compute polarizabilities for highly polarizable systems whose exact wave function is more diffuse than the one of $\mathrm{He}$ and $\mathrm{H}$, we selected the hydride ion as test case. For this system we optimized a five-term Hylleraas-type wave function whose average properties are shown in Table I together with the accurate results obtained in Ref. [26]. Table I also contains the multipole static polarizabilities computed in this paper employing a time step of 0.01 hartree $^{-1}$, and the static polarizability computed by Glover and Weinhold [24]. Comparing the mean values in Table I, one can notice that our five-term wave function gives lower values than the ones obtained in Ref. [26] except for $\left\langle r_{-}\right\rangle$. This fact may explain the underestimation of the $\alpha_{d i p}$ by PT-VMC, that recovers $92(2) \%$ of the accurate value. Nevertheless, this result represents a fairly good estimation of the static dipole polarizability for $\mathrm{H}^{-}$, a quantity that appears difficult to compute even with more complex approaches [27].

As far as PsH is concerned, we computed the autocorrelation functions using two different trial wave functions, including $1\left(\Psi_{T}^{1}\right)$ and $28\left(\Psi_{T}^{28}\right)$ terms [3]. The choice of two trial wave functions to guide the Langevin dynamics was aimed at testing the dependency of $C_{V V}(t)$ on the quality of the wave function itself.

Employing the PT-VMC method and our wave functions for $\mathrm{PsH}$, we computed the autocorrelation functions for three perturbation potentials:

$$
\begin{gathered}
V_{1}=x_{1}+x_{2}-x_{p}, \\
V_{2}=\frac{3\left(x_{1}^{2}+x_{2}^{2}-x_{p}^{2}\right)-\left(r_{1}^{2}+r_{2}^{2}-r_{p}^{2}\right)}{2}, \\
V_{3}=x_{1}^{3}+x_{2}^{3}-x_{p}^{3}-\frac{3\left[x_{1}\left(y_{1}^{2}+z_{1}^{2}\right)+x_{2}\left(y_{2}^{2}+z_{2}^{2}\right)-x_{p}\left(y_{p}^{2}+z_{p}^{2}\right)\right]}{2},
\end{gathered}
$$




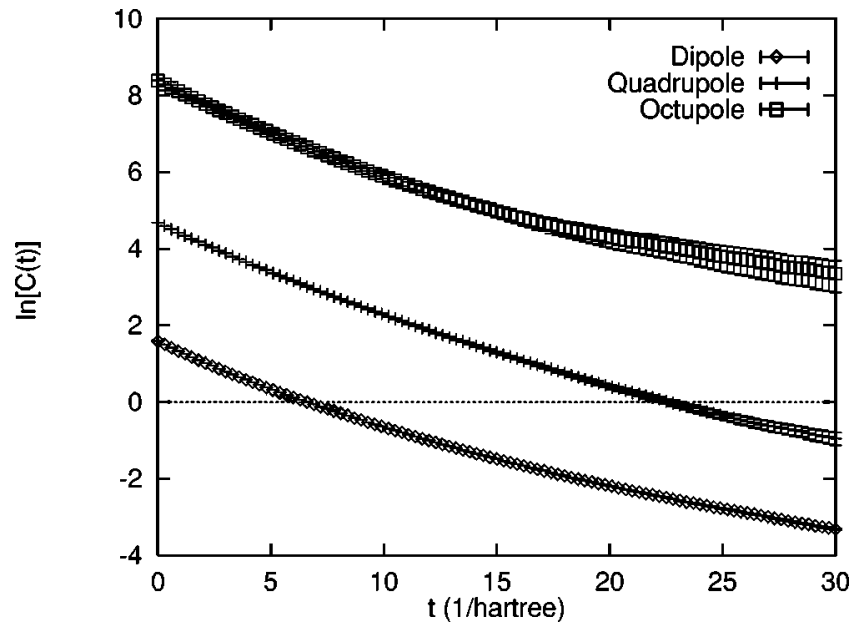

FIG. 1. Logarithm of the correlation functions of the perturbing potentials.

where the subscripts 1 and 2 indicate the two electrons, while the subscript $p$ indicates the positron. These potentials are the Cartesian forms of the dipole, quadrupole, and octupole moment operators for the $\mathrm{PsH}$ system. Figure 1 shows the averaged correlation functions for $V_{1}, V_{2}$, and $V_{3}$ as obtained by the VMC method employing the 28-term trial wave function. Each value of the correlation functions was computed employing roughly $10^{10}$ configurations. From Fig. 1 one can note the effect at large evolution times of the dispersion of the "trajectories" used to compute the autocorrelation function. This effect makes difficult the reproduction of the long-time regime of these functions due to the exponential decay and the roughly constant statistical error introduced by the method. Moreover, the statistical error strongly depends on the perturbation potential whose autocorrelation function is computed, i.e., more specifically on the dispersion of its mean value over the $\Psi_{T}^{2}$ distribution.

The results for the static multipole polarizabilities, i.e., for $\omega=0$, computed with both trial-wave-functions, are shown in Table II. While for the dipole polarizabilities there is a good agreement between the two values, larger differences are present for the higher multipole polarizabilities. This fact is an indication of the different accuracy of the two functions in approximating the exact wave function at large distances from the nucleus. In fact it can be shown that if one approximates the autocorrelation functions taking care only of the excitation to the first state of the appropriate symmetry, the autocorrelation function is proportional to $\left\langle V_{i}^{2}\right\rangle-\left\langle V_{i}\right\rangle^{2}$, where $V_{i}$ is the perturbing potential. Comparing the dipole results with the value obtained by Le Sech and Silvi [6],

TABLE II. Static multipole polarizabilities for the ground-state ${ }^{2,1} S$ of the PsH computed with 1-term $\left(\Psi_{T}^{1}\right)$ and 28-term $\left(\Psi_{T}^{28}\right)$ wave functions. All values are in atomic units.

\begin{tabular}{cccc}
\hline \hline$\Psi_{T}$ & $\alpha_{\text {dip }}$ & $\alpha_{\text {quad }}$ & $\alpha_{\text {oct }}$ \\
\hline$\Psi_{T}^{1}$ & $43.66(3)$ & $972.7(2)$ & $39178(32)$ \\
$\Psi_{T}^{28}$ & $42.99(4)$ & $876.9(3)$ & $34848(71)$ \\
\hline \hline
\end{tabular}

TABLE III. Computed dispersion coefficients. All values are in atomic units.

\begin{tabular}{lccc}
\hline \hline & $C_{6}$ & $C_{8}$ & $C_{10}$ \\
\hline $\mathrm{H}-\mathrm{H}$ & 6.480 & 125.23 & 3318.2 \\
& $6.499^{\mathrm{a}}$ & $124.39^{\mathrm{a}}$ & $3285.8^{\mathrm{a}}$ \\
$\mathrm{H}-\mathrm{He}$ & 2.813 & 41.671 & 866.33 \\
& $2.821^{\mathrm{a}}$ & $41.836^{\mathrm{a}}$ & $871.54^{\mathrm{a}}$ \\
$\mathrm{He}-\mathrm{He}$ & 1.454 & 13.880 & 177.01 \\
& $1.461^{\mathrm{a}}$ & $14.117^{\mathrm{a}}$ & $183.69^{\mathrm{a}}$ \\
$\mathrm{H}-\mathrm{PsH}$ & 40.30 & 2596.1 & 86292 \\
$\mathrm{He}-\mathrm{PsH}$ & 15.718 & 950.80 & 23490 \\
\hline \hline
\end{tabular}

${ }^{\mathrm{a}}$ Reference [25].

again the large difference of the computed polarizabilities is apparent. As a final check for this problem, we computed the energy of the PsH when immersed in a weak static electric field $F$ by means of standard DMC simulations adding the linear potential $F\left(x_{1}+x_{2}-x_{p}\right)$. To make our simulations stable, i.e., to avoid the dissociation of the $\mathrm{PsH}$, we truncated the effect of the linear potential at $\left|x_{i}\right|=15$ bohr. We fitted the DMC results by means of the simple polynomial $a$ $-\alpha_{\text {dip }} F^{2} / 2$, where $\alpha_{d i p}$ is the static dipole polarizability, obtaining $\alpha_{\text {dip }}=42.3(8)$ a.u. We believe that this result, statistically indistinguishable from the $\alpha_{\text {dip }}$ obtained by the PTVMC method, gives the definitive answer to the problem of the PsH polarizability. Nevertheless, the discrepancy between our PT-VMC and DMC $\alpha_{d i p}$ and the one computed by Le Sech and Silvi [6] remains puzzling. In our experience [3], to compute the matrix elements they needed, millions of configurations must be used even for systems like PsH to avoid being fooled by a false convergence. Unfortunately, Le Sech and Silvi did not report any information about the number of configurations they used to compute the integrals, so we cannot judge the numerical accuracy of their results.

An attempt to estimate the total accuracy of our $\alpha$ results can be made comparing the polarizability values obtained by the two wave functions. These differ by $10 \%$ at most, a value that we feel might give a conservative estimate of the relative errors for the higher multipolar fields.

As stated previously, although dynamical polarizabilities are interesting on their own, they represent the basis to compute van der Waals dispersion coefficients for the interaction between different systems. Therefore, following Ref. [25], we present the calculation of the $C_{6}, C_{8}$, and $C_{10}$ dispersion coefficients between $\mathrm{H}, \mathrm{He}$, and $\mathrm{PsH}$ as a first effort to obtain accurate information on the interaction between positronic systems and ordinary matter in the framework of the BornOppenheimer approximation and second-order perturbation theory.

Using the fitted parameters for $\mathrm{H}, \mathrm{He}$, and $\mathrm{PsH}$ we computed the coefficients for the interaction between the ordinary systems and between these and $\mathrm{PsH}$. The values are reported in Table III. Since the values for the $\mathrm{H}-\mathrm{H}, \mathrm{H}-\mathrm{He}$, and He-He coefficients are accurately known [25], we use them as a test of the accuracy of our approach: all the values differ from the accurate results by Yan et al. [25] at most by one part over hundreds. 
Comparing the $C_{n}$ 's for the ordinary systems with the ones for the interaction with $\mathrm{PsH}$, it strikes us that these last are more than an order of magnitude larger than the former. These features, due to the larger $\mathrm{PsH}$ polarizability, indicates that positronic systems strongly interact with ordinary matter even at large distances. Unfortunately, nothing can be said about location and depth of the total potential minimum. This strongly depends also on the effect of the repulsion between the positron cloud and the $\mathrm{H}$ and $\mathrm{He}$ nuclei, so that we believe a supermolecule approach is needed. In a previous work [17] we computed the interaction energy between $\mathrm{H}$ and $\mathrm{PsH}$, showing that this system could have a metastable state. Although the dispersion coefficients for the interaction between $\mathrm{He}$ and $\mathrm{PsH}$ are smaller than those for $\mathrm{PsH}$ and $\mathrm{H}$, they might be large enough to give rise to a potential well that could support at least a stable state. If this turns out to be the case, the He-PsH system could be the lightest van der Waals (i.e., bound by means of dispersion forces) stable dimer.

Financial support by the Universita' degli Studi di Milano is gratefully acknowledged. The authors are indebted to the Centro CNR per lo Studio delle Relazioni tra Struttura e Reattivita' Chimica for grants of computer time.
[1] G. G. Ryzhikh, J. Mitroy, and K. Varga, J. Phys. B 31, 3965 (1998).

[2] T. Yoshida, G. Miyako, N. Jiang, and D. M. Schrader, Phys. Rev. A 54, 964 (1996).

[3] M. Mella, G. Morosi, and D. Bressanini, J. Chem. Phys. 111, 108 (1999).

[4] N. Jiang and D. M. Schrader, J. Chem. Phys. 109, 9430 (1998); Phys. Rev. Lett. 81, 5113 (1998).

[5] K. Strasburger, J. Chem. Phys. 111, 10555 (1999).

[6] C. Le Sech and B. Silvi, Chem. Phys. 236, 77 (1998).

[7] D. C. Clary, J. Phys. B 9, 3115 (1976).

[8] Y. K. Ho, Phys. Rev. A 34, 609 (1986).

[9] A. M. Frolov and V. H. Smith, Jr., Phys. Rev. A 56, 2417 (1997).

[10] M. T. McAlinden, F. G. R. S. MacDonald, and H. R. J. Walters, Can. J. Phys. 74, 434 (1996).

[11] S. Sur, S. K. Adhikari, and A. S. Ghosh, Phys. Rev. A 53, 3340 (1996).

[12] M. Comi, G. M. Prosperi, and A. Zecca, Nuovo Cimento D 2, 1347 (1983).

[13] P. K. Biswas and A. S. Ghosh, Phys. Lett. A 223, 173 (1996).

[14] P. K. Biswas and S. K. Adhikari, J. Phys. B 31, L315 (1998).

[15] P. Chaudhuri, S. K. Adhikari, B. Talukdar, and S. Bhatta- charyya, Eur. Phys. J. D 5, 217 (1999).

[16] B. Nath and C. Sinha, Eur. Phys. J. D 6, 295 (1999).

[17] M. Mella, G. Morosi, and D. Bressanini, J. Chem. Phys. 112, 1063 (2000).

[18] G. Lamm and A. Szabo, J. Chem. Phys. 72, 3354 (1980).

[19] M. Caffarel and O. Hess, Phys. Rev. A 43, 2139 (1991).

[20] M. Mella, G. Morosi, D. Bressanini, and S. Elli, J. Chem. Phys. 113, 6154 (2000).

[21] B. L. Hammond, W. A. Lester, Jr., and P. J. Reynolds, Monte Carlo Methods in Ab Initio Quantum Chemistry, 1st ed. (World Scientific, Singapore, 1994).

[22] M. Caffarel, M. Rérat, and C. Pouchan, Phys. Rev. A 47, 3704 (1993).

[23] G. Migliavacca, Thesis, University of Milan, 1998 (unpublished).

[24] R. M. Glover and F. Weinhold, J. Chem. Phys. 65, 4913 (1976).

[25] Z. Yan, J. F. Babb, A. Dalgarno, and G. W. F. Drake, Phys. Rev. A 54, 2824 (1996).

[26] T. Koga, Y. Kasai, and A. J. Thakkar, Int. J. Quantum Chem. 46, 689 (1993).

[27] D. Spelsberg, T. Lorenz, and W. Meyer, J. Chem. Phys. 99, 7845 (1993). 